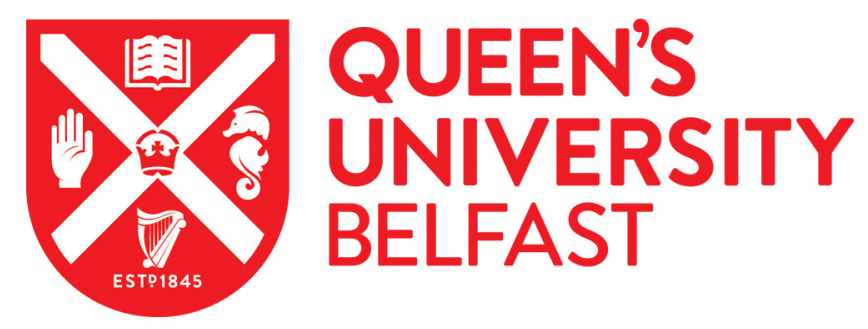

\title{
Fractional exhaled nitric oxide as a predictor of response to inhaled corticosteroids in patients with non-specific respiratory symptoms and insignificant bronchodilator reversibility: a randomised controlled trial
}

Price, D., Buhl, R., Chan, A., Freeman, D., Gardener, E., Goodley, C., Gruffydd-Jones, K., McGarvey, L., Ohta, K., Ryan, D., Syk, J., Chuan Tan, N., Tan, T., Thomas, M., Yang, S., Raju Konduru, P., Ngantcha, M., Stagno d'Alcontres, M., \& Lapperre, T. (2018). Fractional exhaled nitric oxide as a predictor of response to inhaled corticosteroids in patients with non-specific respiratory symptoms and insignificant bronchodilator reversibility: a randomised controlled trial. The Lancet Respiratory Medicine, 6(1), 29-39. https://doi.org/10.1016/S22132600(17)30424-1

Published in:

The Lancet Respiratory Medicine

Document Version:

Peer reviewed version

Queen's University Belfast - Research Portal:

Link to publication record in Queen's University Belfast Research Portal

\section{Publisher rights}

Copyright 2017 Elsevier.

This manuscript is distributed under a Creative Commons Attribution-NonCommercial-NoDerivs License

(https://creativecommons.org/licenses/by-nc-nd/4.0/), which permits distribution and reproduction for non-commercial purposes, provided the author and source are cited.

\section{General rights}

Copyright for the publications made accessible via the Queen's University Belfast Research Portal is retained by the author(s) and / or other copyright owners and it is a condition of accessing these publications that users recognise and abide by the legal requirements associated with these rights.

Take down policy

The Research Portal is Queen's institutional repository that provides access to Queen's research output. Every effort has been made to ensure that content in the Research Portal does not infringe any person's rights, or applicable UK laws. If you discover content in the Research Portal that you believe breaches copyright or violates any law, please contact openaccess@qub.ac.uk. 


\section{FeNO as predictor of response to inhaled corticosteroids in}

\section{patients with non-specific respiratory symptoms and}

\section{insignificant bronchodilator reversibility; a randomised}

\section{controlled trial}

Prof David B Price, FRCGP1,2*, Prof Roland Buhl, MD³, Dr Adrian Chan, MRCP 4 , Dr Daryl Freeman, FRCGP 5 , Ms Elizabeth Gardener, MSc ${ }^{2}$, Dr Clifford Godley, FRGCP ${ }^{6}$, Dr Kevin Gruffydd-Jones, FRGCP ${ }^{7}$, Dr Lorcan McGarvey, MD${ }^{8}$, Prof Ken Ohta, MD ${ }^{9}$, Dr Dermot Ryan, FRGCP10, Dr Jörgen Syk, PhD11,12,13, Dr Ngiap Chuan Tan, FAMS ${ }^{14,15}$, Dr TzeLee Tan, FRCPE ${ }^{16}$, Prof Mike Thomas, $\mathrm{PhD}^{17}$, Dr Sen Yang, MD², Mrs Priyanka Raju Konduru, MS ${ }^{18}$, Mr Marcus Ngantcha, MSc'2, Dr Martina Stagno d'Alcontres, $\mathrm{PhD}^{2}$, Dr Therese S Lapperre, $\mathrm{PhD}{ }^{16,19}$

1. University of Aberdeen, Aberdeen, United Kingdom

2. Observational \& Pragmatic Research Institute, Singapore, Singapore

3. Mainz University Hospital, Mainz, Germany

4. Singapore General Hospital, Singapore, Singapore

5. Mundesley Medical Centre, Norfolk, United Kingdom

6. Avondale Medical Practice, Strathaven, United Kingdom

7. Box Surgery, Box, United Kingdom

8. Queen's University Belfast, Belfast, United Kingdom

9. National Hospital Organization Tokyo National Hospital, Tokyo, Japan

10. Usher Institute of Population Health Sciences and Informatics, Edinburgh, United Kingdom

11. Karolinska Institutet, Stockholm, Sweden

12. Uppsala University, Uppsala, Sweden

13. Academic Primary Health Care Centre, Stockholm, Sweden

14. SingHealth Polyclinics, Singapore, Singapore 
15. Duke-NUS Medical School, Singapore, Singapore

16. National University of Singapore, Singapore, Singapore

17. University of Southampton, Southampton, United Kingdom

18. Optimum Patient Care, Cambridge, United Kingdom

19. Pulmonary Department, Bispebjerg Hospital, Copenhagen, Denmark

${ }^{*}$ Corresponding author: Prof David B Price, Academic Primary Care, Division of Applied Health Sciences, University of Aberdeen, Polwarth Building, Foresterhill, Aberdeen, UK AB25 2ZD Tel: +65 6802 9724; E-mail: dprice@opri.sg

\section{RESEARCH IN CONTEXT}

\section{Evidence before this study:}

Numerous studies have provided evidence regarding the applications of fractional exhaled nitric oxide (FeNO) measurements in the clinical management of asthma. To identify studies in which FeNO was used in patients with non-specific respiratory symptoms we searched PubMed up until $1^{\text {st }}$ January 2015 for randomised controlled trials which investigated the association between fractional exhaled nitric oxide (FeNO) and response to inhaled or oral steroids in patients with unclear asthma, using search terms: "feno", "asthma", ("chronic respiratory symptoms" OR "unspecific respiratory symptoms" OR "undiagnosed asthma" OR "non-specific respiratory symptoms") and ("steroids" OR "corticosteroids"). The majority of trials were excluded for not evaluating the required association. In the trial by Smith et al, in patients with undiagnosed respiratory symptoms, the group with the highest FeNO (>47 ppb) demonstrated a significantly greater response to four weeks of inhaled corticosteroids (ICS) in all outcomes. A recent study in patients with confirmed asthma, by Malinovschi et al, found that FeNO levels were associated with improved asthma control after ICS treatment in patients with intermediate ( $\geq 25$ and $<50 \mathrm{ppb}$ ) and high FeNO ( $\geq 50 \mathrm{ppb})$, compared to normal levels $(<25 \mathrm{ppb})$. 


\section{Added value of this study}

The previous trial of FeNO in a non-specific respiratory symptoms (NSRS) patient population has weaknesses mainly due to the relatively small sample size $(n=52)$ and study design (single-blind, fixed-sequence design with placebo period followed by treatment period). To our knowledge, the current trial is the largest randomised placebo-controlled trial using FeNO in an NSRS patient population to date, and therefore offers well-powered evidence. The NSRS population assessed in this trial is a difficult-to-manage patient population with insignificant bronchodilator reversibility. The results were consistent with previous findings in both asthma and undiagnosed asthma, with a clear association between high FeNO and a greater likelihood to respond to inhaled corticosteroids.

\section{Implications of all the available evidence}

This study provides strong support for the use of FeNO measurement in primary care, to inform ICS prescribing decisions in patients with non-specific respiratory symptoms, unclear asthma and insignificant bronchodilator reversibility. This data supports a targeted individualised approach in the clinical management of chronic airway diseases.

\section{ABSTRACT}

\section{Background:}

Chronic non-specific respiratory symptoms (NSRS) are difficult to manage. This trial evaluated the association between FeNO and the response to Inhaled corticosteroids (ICS) in NSRS patients.

Methods: A double-blind randomised controlled trial in undiagnosed patients, aged 18-80, with cough, wheeze and/or dyspnoea and $<20 \%$ bronchodilator reversibility, 4-week treatment period with ICS (QVAR $80 \mathrm{mcg}$, two puffs twice per day, equivalent to $400 \mathrm{mcg}$ beclomethasone dipropionate) or placebo. Stratified randomisation was carried out within categories of baseline FeNO - normal ( $\leq 25 \mathrm{ppb})$, intermediate ( $>25-<40 \mathrm{ppb})$, and high $(\geq 40$ 
ppb) - allocation. Generalised linear modelling assessed FeNO as a predictor of response, estimating an interaction effect between FeNO and treatment, on change in ACQ7. Logistic regression assessed FeNO and clinical opinion of asthma status as predictors of response.

Findings: 294 patients were randomised (148 ICS, 146 placebo). Following exclusions due to protocol violations 214 patients were analysed (114 ICS, 100 placebo). Treatment effects were significant for high FeNO (mean change in ACQ7, $0.49[95 \% \mathrm{Cl}, 0.14,0.84]$ ), nonsignificant for normal and intermediate FeNO $(0.10[95 \% \mathrm{Cl},-0.24,0.44]$ and $0.25[-0.10,0.61]$ : $\mathrm{P}=0.044$ for interaction). FeNO $>50 \mathrm{ppb}$ was associated with greater odds of improvement in cough on the visual analogue scale (odds ratio, 2.37 [1.01, 5.55]), while an affirmative clinical opinion of asthma was not predictive $(0.92[0.47,1.82])$. This study is registered on ClinicalTrials.gov (number: NCT02294279).

Interpretation: FeNO may predict response to ICS in patients with NSRS, while clinical opinion is not predictive.

Funding: Partially funded by Circassia, formerly Aerocrine.

\section{INTRODUCTION}

The European Asthma Research and Innovation Partnership rated asthma diagnosis as the most important area requiring further research.(1) Respiratory symptoms including cough and breathlessness are a common presentation in the primary care setting, and can be difficult to manage(2) - particularly when symptoms are non-specific. Treatment decisions are even more difficult if the potential diagnosis is not supported by typical criteria, e.g. patient has suspected asthma but does not show reversibility to short-acting beta agonists (SABA). Such symptom patterns, as well as unexplained chronic cough, cause significant impairments in quality of life. Effective assessment and treatment approaches are needed.(3)

Inhaled corticosteroids (ICS) are often prescribed, however, the response to ICS varies widely within patients with confirmed asthma,(4) which may also be the case for patients with respiratory symptoms without clear asthma. Identifying patients most likely to respond to ICS 
would minimise inappropriate treatment and limit side effects and unnecessary healthcare costs. $(5,6)$ One potential strategy to target this problem is to assess fractional exhaled nitric oxide (FeNO) in addition to the current standard of care when informing ICS prescribing decisions. FeNO has been identified as a marker for type-2 allergic airway inflammation(7) in patients with asthma,(8) and has been shown to be a reliable predictor of responsiveness to ICS in patients with asthma.(8-11) A type-2 pattern of airway inflammation, involving lymphoid cells, T-helper 2 cells, tissue eosinophilia, and IgE production, (12) may be evident in patients without an established asthma diagnosis, opening the potential for FeNO-guided treatment in this population. On this strategy, clinical guidelines are divided: NICE recommends FeNO measurement to guide diagnosis and management of asthma, (13) while GINA states that FeNO cannot yet be recommended, based on the current body of evidence.(14) While this inconsistent guidance exists for patients with asthma, due to limited evidence, there is even less data for patients without clear asthma.

Among the limited evidence in the population without a clear asthma diagnosis, one study found that an elevated baseline FeNO level (>47 ppb) in patients with previously undiagnosed respiratory symptoms was predictive of an ICS response. In this study, steroid response was defined as change in symptoms, peak flows, spirometry, or airway hyperresponsiveness to adenosine based on established guidelines and recommendation.(15) However, this was a small, single-blind study and these results alone are not entirely persuasive.

Clinicians rely on a range of measures including FeNO, spirometry, blood eosinophils and their own clinical judgement when assessing patients but there is little information on what is most useful in guiding ICS treatment in patients with undiagnosed respiratory symptoms. To this end we carried out a randomised placebo-controlled trial to investigate the value of FeNO, and other baseline measurements, in predicting a clinical response to 4-weeks of treatment with ICS, in a sample of patients with non-specific respiratory symptoms without asthma and insignificant bronchodilator reversibility. 


\section{METHODS}

\section{Study design}

This was a six-week randomised, multi-centre, double-blind, placebo-controlled study, at primary care centres and hospital sites in the UK and Singapore. Patient enrolment began on the 4th of February 2015 with the last patient completing the study on the $26^{\text {th }}$ August 2016. The study consisted of a two-week assessment period, for screening and measurement of baseline variables, followed by a four-week treatment period. Participants were randomised at the end of the assessment period, to either the treatment arm (extrafine ICS - QVAR 80 mcg Inhalation Aerosol, two puffs twice per day, equivalent to standard $400 \mathrm{mcg}$ beclomethasone dipropionate) or the control arm (placebo - same regimen of two puffs twice per day, with inactive ingredient). The active study drug and matching placebo were provided by TEVA RESPIRATORY, LLC Teva Pharmaceuticals Europe BV, Piet Heinkade 107, 1019 GM Amsterdam, the Netherlands. The study sample population consisted of patients (aged 18-80 years) with non-specific, persistent respiratory symptoms (cough and/or wheeze and/or chronic dyspnoea for $\geq 6$ weeks before screening), with no previous diagnosis of asthma.

If patients displayed a forced expiratory volume in the first second $\left(\mathrm{FEV}_{1}\right)<90 \%$ predicted at screening, they also needed to show an insignificant reversibility predefined as $\mathrm{FEV}_{1}<20 \%$ to short-acting beta-agonists (SABA) at visit 1 or within the previous year. Subjects who were ever diagnosed with asthma or any other significant chronic respiratory disease, and/or who had received treatment with oral, inhaled or systemic corticosteroids, a leukotriene modifier or long-acting beta agonist within four weeks prior to the first study visit, were excluded. The full study inclusion and exclusion criteria can be found in Supplementary table 1.

The primary objective was to determine whether the response to treatment (extrafine ICS) varies between FeNO groups by considering the change in ACQ scores from baseline to follow-up. For this objective, response to treatment was measured in terms of change in Asthma Control Questionnaire (ACQ7) from baseline. The ACQ7 was selected as there is 
no appropriate endpoint for patients with non-specific respiratory symptoms. The steering committee chose the ACQ7 a priori as this was believed to be the closest response assessment to date for this patient population. Management of NSRS patients in routine clinical care is similar to the management of patients suspected for asthma, therefore a pragmatic study approach was adopted to reflect real-life clinical care.

The secondary objectives were to investigate the relationship between FeNO and treatment response, in terms of changes from baseline in symptom and cough severity scores (each measured in millimetres on a visual analogue scale [VAS]), and changes in spirometry measurements. The first exploratory objective was to investigate interaction effects between FeNO and treatment arm, on the following endpoints: Global Evaluation of Treatment Effectiveness (GETE) scale (16) and EuroQol 5 Dimensions Questionnaire (EQ-5D-3L).(17) The second exploratory objective was to evaluate the following factors as predictors of clinical response; FeNO, spirometry, peripheral blood eosinophil count and clinical judgement of asthma diagnosis. For this objective, clinical responses to treatment were defined in two ways - as a decrease in ACQ7 $\geq 0.5$, and a decrease in visual cough score of $\geq 20 \mathrm{~mm}$.

The study and all amendments were approved by the National Research Ethics Service Committee Yorkshire \& the Humber - South Yorkshire (14/YH/0129), for UK sites. For Singapore sites, the study was approved by the Parkway Independent Ethics Committee (PIEC/2014/028) and SingHealth Centralised Institutional Review Board (CIRB/2014/2052). This study is registered on ClinicalTrials.gov (NCT02294279) and on the Health Research Authority NHS register (Eudract number 2014-000051-90). The study was conducted in full accordance with the International Conference on Harmonisation (ICH) Good Clinical Practice (GCP) Consolidated Guideline (E6) requirements of the ICH E2D guideline (Post-Approval Safety Data Management: Definitions and Standards for Expedited Reporting), the principles of the Declaration of Helsinki and the EU Clinical Trials Directive (2001/20/EC) as well as with any other applicable national and local laws and regulations. 


\section{Study procedures}

Three visits were involved in the study procedures: visit 1, during which patients were screened according to study criteria and invited to participate; visit 2, during which eligibility for further study participation was confirmed and patients were randomised to ICS or placebo, and; visit 3 (end of study visit), after the four-week treatment period, during which the final outcome measurements were assessed. Supplementary table 2 provides details of study procedures and assessments. The two-week assessment period (between visits 1 and 2 ) had a window of $+/-7$ days and the four-week treatment period had a window of $+/-2$ days (between visits 2 and 3 ). At visit 1 , baseline measurements were performed for eligible participants, including: FeNO (measured using NIOX VERO [Circassia]); Asthma Control Questionnaire (ACQ7); perception of asthma symptoms measured on a 0-100 horizontal visual analogue scale (VAS Symptom), where 0 is not bothersome at all and 100 is extremely bothersome; severity of cough measured on a 0-100 vertical visual analogue scale (VAS Cough), where 0 is no cough and 100 is worse cough ever; $\mathrm{FEV}_{1} ; \mathrm{FEV}_{1} \%$ predicted; forced vital capacity (FVC); FVC\% predicted; FEV 1 /FVC; peak expiratory flow (PEF); peripheral blood eosinophils (count x 109/L); GETE, and; EQ-5D-3L. In addition, the patients' clinicians were asked to give a yes or no response to the question, "In your clinical opinion, is the patient asthmatic?".

At visit 2, patients were asked if their non-specific respiratory symptoms had persisted since the previous visit. If symptoms were not persistent, the patient was excluded. Patients were asked about medications taken between visit 1 and visit 2: if any were prohibited medications for the study (oral, inhaled or systemic corticosteroids, leukotriene modifiers, or long-acting beta agonists), the patient was excluded. Finally, patients were asked if they experienced any adverse events between visit 1 and visit 2 (before receiving the study drug or placebo): the patient was excluded if they experienced one or more adverse events.

Following confirmation of informed consent by eligible participants, stratified randomisation was carried out, within predefined categories of baseline FeNO (normal, $\leq 25 \mathrm{ppb}$; intermediate, >25 - <40 ppb; high, $\geq 40$ ppb). $(15,18)$ 
Block randomisation was performed by in-house software using blocks of size 6 with a 1:1 ratio of active to placebo and were stratified by FeNO category. The system kept track of all kit numbers and respective status, whenever randomisation request was made the appropriate kit number was delivered to end user. All the information was concealed securely in a server and none of the study team had access to it. All data was captured electronically, using the Enterprise system of OpenClinica software.(19) Unblinding was performed after data quality checks and database lock and once primary and secondary analyses were complete.

\section{Study endpoints}

The primary endpoint was to analyse the interaction between baseline FeNO and the treatment effect in ACQ change compared to placebo. The primary comparison was the overall interaction between treatment groups to understand if FeNO levels among treatment groups can modify the change in ACQ7 score. Interaction analysis was used to determine whether the effect or, more specifically, the response to the treatment, varies between the FeNO groups.

Secondary endpoints, for which interaction effects were also estimated, included changes in: VAS Symptoms; VAS Cough, and; lung function $\left(\mathrm{FEV}_{1}, \mathrm{FEV}_{1} \%\right.$ predicted, $\mathrm{FVC}, \mathrm{FVC} \%$ predicted, PEF). Exploratory endpoints included changes from baseline in GETE and EQ-5D$3 \mathrm{~L}$.

\section{Statistical analysis}

A Per-Protocol set, in which all primary and secondary analyses were carried out, was prepared by applying the following exclusions to the randomised study sample: noncompletion of the primary endpoint; non-compliance to treatment (ascertained by patient report), and study visits made outside the predefined visit windows. A Main-Analysis set was also created, where the study window exclusion criteria was not applied.

Based on $80 \%$ power for detecting a treatment/FeNO interaction effect in the primary analysis, a required sample size of 264 patients was computed. Baseline summary statistics were 
computed and compared between treatment arms, using Mann-Whitney and chi-square tests. The primary analysis used Generalised Linear Models to estimate the treatment effects, in terms of change in ACQ7, in each of the three baseline FeNO categories. An interaction was then tested, between treatment arm and baseline FeNO, on change in ACQ7 and ACQ6. This was done using another Generalised Linear Model, with FeNO centred on the mean. The coefficient of the interaction effect was obtained from the model, with $95 \%$ confidence intervals (CI). The interaction effect was interpreted as the change in treatment effect on the outcome when baseline FeNO increases. Similar models were repeated to estimate interaction effects on the secondary and exploratory endpoints. In all models, there was adjustment by smoking status (a potential confounder). A multiple linear regression was undertaken to evaluate the difference in gradients. The covariates added in this model will include the baseline value of the ACQ7 score.

In the Main Analysis set (and in the extrafine ICS arm only), predictors of clinical response to treatment were investigated, in terms of ACQ7 (decrease $\geq 0.5$ ) and VAS Cough (decrease $\geq$ $20 \mathrm{~mm}$ ). Potential predictors were tested in univariable logistic regression models and, if statistically significant and routinely taken in clinical practice, were included in a multivariable model which included baseline FeNO as a covariate.

All statistical analyses were performed using SAS version 9.3.(20)

\section{Role of funding source}

This was an investigator-initiated trial, with Prof David B Price as Chief Scientist and Observational \& Pragmatic Research Institute as the sponsor. Partial study funding was provided by Circassia, formerly Aerocrine, in the form of an unrestricted grant. Circassia provided NIOX machines for FeNO measurement on loan for the duration of the trial. Circassia did not contribute to the trial in any other way: there was no involvement in the trial design or execution of the trial such as data collection, analysis or interpretation of the data, or any aspect pertinent to the trial or the content of the manuscript. In addition, TEVA 
Pharmaceuticals did not provide funding, but provided the study drugs, QVAR and matching placebo. TEVA did not contribute to the trial in any other way: there was no involvement in the trial design or execution of the trial such as data collection, analysis or interpretation of the data, or any aspect pertinent to the trial or the content of the manuscript.

\section{RESULTS}

\section{Patients}

Of the 517 patients screened at 26 UK primary care centres and 3 Singapore centres, 294 patients were randomised after applying inclusion and exclusion criteria; 148 to the extrafine ICS arm and 146 to the placebo arm. Following exclusions for non-completion of the primary endpoint and non-compliance to treatment, 259 patients were included in the Main-Analysis set. Following further exclusions for protocol violation, the Per-Protocol (PP) set consisted of 214 patients - 114 in the extrafine ICS arm and 100 in the placebo arm (Figure 1). The main reason for ineligibility to the PP set was that the study visits were outside the study window.

In the Per-Protocol set, mean ages were 50.0 (standard deviation [SD], 15.5) and 47.7 years $(S D, 17.0)$ in the extrafine ICS and placebo arms, respectively; over half of the sample were women, and $73 / 114=64.0 \%$ and $73 / 100=73.0 \%$ were non-smokers, in the extrafine ICS and placebo arms, respectively (Table 1). The proportions of patients predicted to be asthmatic by clinicians were $62 / 114=54.9 \%$ in the extrafine ICS arm and $60 / 100=60.0 \%$ in the placebo arm. Patients in whom reversibility was determined at baseline, mean scores (computed as difference in $\mathrm{FEV}_{1}$ before and after salbutamol multiplied by 100$)$ were $2.37 \%(\mathrm{SD}, 0.84)$ and $2.47 \%(\mathrm{SD}, 0.74)$ in the extrafine ICS and placebo arms, respectively. Only 4 patients (2 in the extrafine ICS arm and 2 in the placebo arm) were active or ex-smokers and had spirometry values that would meet a COPD diagnosis. Baseline characteristics by FeNO category can be found in Supplementary table 3. Approximately $40 \%$ of patients reported all three symptoms of cough, dyspnoea and wheeze. Cough was the most prevalent symptom, reported by over $80 \%$ of patients. The majority of patients had an $\mathrm{FEV}_{1} \%$ predicted more than $90 \%(63 / 114$ 
$=55.3 \%$ in the ICS group and $63 / 100=63 \%$ in the placebo group) and those with less than $90 \%, 5 / 88=5.7 \%$ had $\geq 15 \% \mathrm{FEV}_{1}$ reversibility (Supplementary table 4 ).

\section{Primary analysis}

We observed a significant interaction between baseline FeNO and treatment arm 0.071 (95\% $\mathrm{Cl}, 0.002,0.139)$ ), suggesting the size of treatment response depended on the level of FeNO. For every $10 \mathrm{ppb}$ increase in baseline FeNO, the change in ACQ7 was 0.071 greater in the extrafine ICS arm compared to the placebo arm (Table 2). From baseline to the end of treatment period, ACQ7 improved by $0.29(95 \% \mathrm{CI}, 0.08-0.49)$ in the extrafine ICS arm compared with the placebo arm. This association was adjusted by baseline FeNO and smoking status. Within baseline FeNO categories, the adjusted treatment effect was statistically significant and clinically relevant in the high FeNO category ( $\geq 40 \mathrm{ppb}$ ), with a mean change in ACQ7 of $0.49(95 \% \mathrm{Cl} ; 0.14,0.84)$, with no statistically significant treatment effects in the normal $(\leq 25 \mathrm{ppb})(0.10(95 \% \mathrm{Cl},-0.24,0.44))$ and intermediate $(>25-<40 \mathrm{ppb})(0.25$ $(95 \% \mathrm{Cl},-0.10,0.61))$ FeNO categories. This was also found when ATS FeNO categorisation was used (Supplementary table 5). The sensitivity analysis further strengthened the association between FeNO and the treatment arm. There was a significant improvement in outcome as FeNO decreases for extrafine ICS compared to placebo prescribed patients $(p=0.024)($ Table 2$)$.

The number of patients showing a clinically important change with ACQ $(\geq 0.5)$ was higher in patients receiving ICS $(80 / 114=70.2 \%)$ treatment compared to those taking placebo $(58 / 100=58.0 \%)($ Table 3$)$.

The interaction between baseline FeNO and treatment arm when ACQ6 was used, was similar to that seen with the ACQ7 (Table 4, Supplementary figure 1). Stratification of the ACQ6 score by FeNO levels found a positive association in the higher FeNO group between ACQ6 and treatment group $0.53(95 \% \mathrm{Cl}, 0.12-0.93)$ (Supplementary table 6). There was a decrease 
in the outcome value of ACQ6 in the ICS group compared with the placebo group after adjustment for smoking and baseline value $(0.076(95 \% \mathrm{Cl},-0.004,0.155))($ Table 4$)$.

\section{Secondary analyses}

There were significant interactions between baseline FeNO and treatment arm, in terms of change in VAS Cough $(3.406(95 \% \mathrm{Cl}, 0.707,6.105))$ and $\mathrm{FEV}_{1}(0.021(95 \% \mathrm{Cl}, 0.005,0.036))$ (Table 5). For the remaining spirometry measurements and for VAS Symptom, the interaction effects were not significant (Table 5).

\section{Exploratory analysis}

The interaction effects for the exploratory endpoints can be seen in Supplementary table 7. In these analyses, there was a significant interaction effect for change in GETE (OR 4.004 (95\% Cl, 1.623, 9.876)), but not for EQ-5D-3L (OR 0.999 (95\% Cl, 0.990, 1.008)).

Of the baseline measurements tested in prediction models, only a report of no additional treatment being taken for their non-specific respiratory symptoms at study enrolment was associated with clinical improvement of ACQ7 (decrease $\geq 0.5$ ), in patients receiving extrafine ICS. Those who did not have additional treatments were over twice as likely to show clinical improvement in ACQ7 compared to those who did (OR, 2.22; 95\% Cl, 1.06-4.76) (Figure 2).

In the model for clinical improvement in cough (defined as a VAS decrease $\geq 20 \mathrm{~mm}$ ), the following baseline measures were significantly predictive in unadjusted models: FeNO (continuous and binary [ $>50 \mathrm{ppb}]$ ), blood eosinophil count and report of dyspnoea symptoms. In the best adjusted model, dyspnoea and FeNO were retained as significant predictors. For every 10ppb increase in baseline FeNO, the odds-ratio of clinical improvement in VAS cough was $1.17(95 \% \mathrm{Cl} ; 1.02-1.34)$ (Figure 2). We observed a positive correlation between improvement in FeNO and ACQ7 scores, EuroQol-5, FEV 1 and VAS cough (Supplementary figure 2). 
Increasing blood eosinophil counts showed a similar trend for improvement in VAS cough or ACQ7 score as did increasing FeNO levels (Supplementary figure 3 and 4).

A summary of adverse events during the study can be found in Table 6 . 


\section{DISCUSSION}

This was a double-blind randomised controlled trial to explore the utility of FeNO in predicting response to four weeks of treatment with inhaled corticosteroids in patients with undiagnosed, non-specific respiratory symptoms. A higher baseline FeNO was associated with a greater likelihood of a positive response to ICS in terms of ACQ7, severity of cough, FEV 1 and GETE. The exploratory analysis found that baseline FeNO was a better predictor of clinical improvement in VAS Cough $(\geq 20 \mathrm{~mm})$ than peripheral blood eosinophils, and that neither $\mathrm{FEV}_{1} \%$ predicted nor clinical opinion of asthma were associated with response to treatment.

These findings build on previous evidence of the utility of FeNO in asthma trials, as well as the limited evidence observed in patients with non-specific respiratory symptoms. In particular, a similar study by Smith et al (15), with a fixed-sequence design rather than parallel arms, also observed associations between baseline FeNO and response to ICS in patients with undiagnosed respiratory symptoms. In this trial, a cut-off for FeNO of $>47 \mathrm{ppb}$ was associated with a predefined minimum increase in $\mathrm{FEV}_{1}$ of $12 \%$ or more. However, the limitations of this study were the small patient number $(n=52)$, the single-blind design, fixed-sequence design with a placebo period followed by treatment.

In the current study, we observed a statistically significant improvement in ACQ7, which reached clinical significance in patients with a high FeNO signal ( $\geq 40 \mathrm{ppb})$. Among patients with non-specific respiratory symptoms, high FeNO levels were able to identify ICS responsive patients. An observational study by Martin et al also reported that FeNO readings were accurate at predicting patient response to ICS.(21) Our data complement these findings using a larger study population further supporting the use of FeNO measurement in patients with non-specific respiratory symptoms potentially responsive to ICS therapy. This would avoid the prescription of unnecessary ICS therapy in patients with low FeNO who are unlikely to benefit from this treatment, and encourage further investigation of the symptoms ensuring more accurate and effective diagnosis and treatment. This finding was further strengthened by the 
statistically significant interaction effect seen between FeNO and ICS treatment. For every 10ppb increase in baseline FeNO, the change in ACQ7 from baseline was $0.071(95 \% \mathrm{Cl}$, $(0.002,0.139)$ greater in those treated with ICS compared to placebo

FeNO also had a strong association with improvement in cough severity. Higher FeNO values were associated with significantly greater odds of a clinical response defined as an improvement of $\geq 20 \mathrm{~mm}$ on the visual analogue scale for cough symptoms. In this trial, the improvement of $20 \mathrm{~mm}$ or more exceeds the minimal important difference suggested in the literature.(22) We observed that patients who did not take any additional therapies for their non-specific respiratory symptoms at study enrolment and who were randomised in the ICS treatment arm, improved clinically with a decrease in ACQ7 of $\geq 0.5$ and were over twice as likely to show clinical improvement in ACQ7 compared to those who did receive additional treatment. We found that there was a greater proportion of patients with normal FeNO values that spontaneously improved compared to those with intermediate or high FeNO. This was likely due to a higher proportion of patients presenting with normal FeNO not suffering from asthma. Although further diagnostics beyond the clinical history and baseline study procedures were not carried out during this study to confirm or exclude an asthma diagnosis, clinical judgement of diagnosis was completely non-predictive of response to ICS versus placebo.

The study population had relatively low levels of symptom severity as measured by ACQ7, which may have influenced the magnitude of the interaction effect of FeNO and treatment on change in ACQ7. This is likely a result of the requirement of no bronchodilator reversibility (< $20 \%$ ) in the study criteria. None of the patients in our study had a reversibility of FEV more $^{2}$ than $19.35 \%$, which is lower than the validated limits as stated by the ATS/ERS guidelines. (23) The sample did not comprise of patients with a pre-existing history of asthma, for which the use of ACQ7 is recommended. In a population with mild or few symptoms, it can be difficult to show improvement in symptoms. Thus, the assay sensitivity of the trial, i.e. its ability to detect effectiveness, may be reduced.(24) The presence of mild symptoms in the study 
population was also thought to be a limiting factor in the study by Smith et al,(15) in which a large proportion of patients with high FeNO did not respond to treatment. It is therefore likely that $A C Q 7$ is not an optimal measure of responsiveness in this population.

We observed that the proportion of male subjects increased in the high FeNO strata. This has been previously reported. Multiple studies have found that FeNO levels are statistically significantly higher in males.(25-28) However, the observed changes were not clinically relevant with changes ranging between 1 and 2-fold.(27) The reason for this association still remains unclear and has not been shown to be influenced by differences in height, vital capacity, or total lung capacity. Future study into whether the observed differences between genders affect the utility of FeNO as a tool for response to therapy will increase accuracy of prescription.

Previous studies have demonstrated that blood eosinophil counts and FeNO offer independent information for the diagnosis of respiratory symptoms (29). Our data show that patients with increasing blood eosinophil counts behave in a similar manner to those with increasing FeNO levels. However, this is an exploratory analysis and further study is needed to confirm the value of combining blood eosinophil counts and FeNO in predicting the response to ICS.

To our knowledge, this is one of the first well-controlled studies investigating the value of FeNO measurement for guiding ICS treatment in a difficult-to-manage patient population with unspecific symptoms. The strengths of this study include its rigorous study design, i.e. randomised, double-blind and placebo controlled, as well as taking place in multiple centres. The study included a treatment period of four weeks, which meets previous recommendations in patients with asthma.(30) The study objectives addressed a patient population that is notably difficult to treat in real-life practice, and for which there is a need for more clinical evidence to guide treatment strategies. Its outcome measures were variables that can be easily measured in routine primary care - FeNO, spirometry and peripheral blood eosinophils - and so its findings are widely implementable. Although the number of patients randomised 
exceeded the target sample size to achieve $80 \%$ power, a limitation of this study is that the size of the Per-Protocol set fell just below this target. Patients were excluded from the PerProtocol analysis mainly because the study visits fell outside the study window. This meant they were no longer taking the assigned treatment thus potentially altering the data collected. The exploratory analysis - which was carried out in the treatment arm only - is particularly affected by reduced power, but the hypotheses generated, concerning the comparison of FeNO and other predictive measures, are valuable for further study.

In terms of clinical practicality and implications, FeNO is a simple and non-invasive tool that can be easily be implemented in routine primary care. The findings of this study suggest that patients with non-specific respiratory symptoms (but no obvious asthma), insignificant bronchodilator reversibility and low FeNO are not likely to respond to ICS and so this treatment could be avoided. ICS are being over prescribed, in recent years regulatory bodies and guidelines have emphasised reducing ICS use where possible. The use of FeNO as a predictor of response to ICS could be an additional tool for health care professionals to optimise patient care. This is a vital implication: once ICS has been initiated, a patient with mild symptoms may appear to have well-controlled disease due to ICS and therefore are unnecessarily exposed to long-term ICS treatment.(31) Clearly, such inappropriate treatment comes at an unnecessary cost and exposes the patient to the potential long-term adverse effects of ICS.(32)

The results of this study also raise a question about what patients mean when they refer to cough or other respiratory symptoms. One previous study examined the use of the word wheeze, demonstrating that it was often cough and other symptoms.(33) It also demonstrated a clear mismatch between doctors and patients concerning the use of these words.

Our findings showed FeNO to have more potential in guiding treatment than either spirometry or eosinophils, although eosinophils were also predictive prior to adjustment by FeNO. Meanwhile, clinical opinion of whether the patient was asthmatic had no relationship with treatment response. This data helps to move forward the research in this difficult-to-manage 
patient population, and with future studies the findings could lead to stronger and refined recommendations for how to manage these patients. Future studies may include more potential predictors, such as a comparison between FeNO and airway hyperresponsiveness - measured often in secondary care - and measurement of outcomes following an extended treatment period beyond 4 weeks.

\section{Conclusion}

FeNO measurement is a simple, non-invasive diagnostic tool that could have a valuable role in identifying a subpopulation of patients with non-specific respiratory symptoms, such as cough, wheeze and shortness of breath, who may benefit from ICS therapy. Our exploratory research suggests FeNO measurement at baseline may be more valuable than spirometry, peripheral blood eosinophils or clinical judgement in predicting treatment response to ICS.

\section{Author contributions}

All authors contributed equally to the study design and protocol, the analysis plan and interpretation of results, the writing of the clinical study report and review of manuscript. In addition to the above, Elizabeth Gardener carried out the statistical analysis. A medical writer assisted in the writing of the manuscript.

\section{Declaration of interests}

DBP has board membership with Aerocrine, Amgen, AstraZeneca, Boehringer Ingelheim, Chiesi, Mylan, Mundipharma, Napp, Novartis, and Teva Pharmaceuticals; consultancy agreements with Almirall, Amgen, AstraZeneca, Boehringer Ingelheim, Chiesi, GlaxoSmithKline, Mylan, Mundipharma, Napp, Novartis, Pfizer, Teva Pharmaceuticals, and Theravance; grants and unrestricted funding for investigator-initiated studies (conducted through Observational and Pragmatic Research Institute Pte Ltd) from Aerocrine, AKL

Research and Development Ltd, AstraZeneca, Boehringer Ingelheim, British Lung Foundation, Chiesi, Mylan, Mundipharma, Napp, Novartis, Pfizer, Respiratory Effectiveness 
Group, Teva Pharmaceuticals, Theravance, UK National Health Service, Zentiva; payment for lectures/speaking engagements from Almirall, AstraZeneca, Boehringer Ingelheim, Chiesi, Cipla, GlaxoSmithKline, Kyorin, Mylan, Merck, Mundipharma, Novartis, Pfizer, Skyepharma, and Teva Pharmaceuticals; payment for manuscript preparation from Mundipharma and Teva Pharmaceuticals; payment for the development of educational materials from Mundipharma and Novartis; payment for travel/accommodation/meeting expenses from Aerocrine, AstraZeneca, Boehringer Ingelheim, Mundipharma, Napp, Novartis, and Teva Pharmaceuticals; funding for patient enrolment or completion of research from Chiesi, Novartis, Teva Pharmaceuticals, and Zentiva; stock/stock options from AKL Research and Development Ltd which produces phytopharmaceuticals; owns $74 \%$ of the social enterprise Optimum Patient Care Ltd (Australia, Singapore, and UK) and $74 \%$ of Observational and Pragmatic Research Institute Pte Ltd (Singapore); and is peer reviewer for grant committees of the Efficacy and Mechanism Evaluation programme, and Health Technology Assessment. RB has received personal fees from AstraZeneca, Boehringer Ingelheim, Chiesi, GlaxoSmithKline, Novartis, Roche, and Teva, as well as grants to Mainz University from Boehringer Ingelheim, GlaxoSmithKline, Novartis, and Roche. KGJ has received personal fees from AstraZeneca, Boehringer Ingelheim, Chiesi, GlaxoSmithKline, Pfizer; grants and personal fees from Napp and Mundipharma, and reduction in cost of FeNo monitor used on practice from Aerocrine. LM has received personal fees from Merck, GlaxoSmithKline; nonfinancial support to attend meetings from Boehringer Ingelheim, Chiesi; and was on the advisory board of and has lectures/speaking engagements from AstraZeneca. Neither MT nor any member of his close family has any shares in pharmaceutical companies. In the last 3 years MT has received speaker's honoraria for speaking at sponsored meetings or satellite symposia at conferences from the following companies marketing respiratory and allergy products: Aerocrine, GSK, Novartis. He has received honoraria for attending advisory panels with; Aerocrine, Boehringer Inglehiem, GSK, MSD, Novartis, Pfizer. He is a member of the BTS SIGN Asthma guideline steering group and the NICE Asthma Diagnosis and Monitoring guideline development group. He has received personal fees from Observational and 
Pragmatic Research Institute Pte Ltd for the work done on this study. EG, MN, MS, PRK, SY are employees of Observational and Pragmatic Research Institute Pte Ltd. CG, DF, DR, JS, KO, TLT, TSL have received personal fees from Observational and Pragmatic Research Institute Pte Ltd for the work done on this study. AC and NCT declare no conflicts of interest.

\section{Acknowledgements}

We thank the study participants, the principal investigators and their staff at the study sites for their contributions. We are grateful to Optimum Patient Care Ltd research team members Latife Hardaker. Miriam Cottle, Francis Appiagyei, Tanith Hjelmbjerg and Elwira Fidziukiewicz for their contributions to site set-up, patient recruitment and conduct of study clinics; Adam Brady and Andrew McLoughlin for their contributions to the data management. We thank the Observational \& Pragmatic Research Institute Pte Ltd research team members Boh Boon Kim and Christina Callan for their support in study coordination and study management. The authors also thank Lisa Law for medical writing support and Carole Nicholls for statistical support. 


\section{References}

1. UK A. European Asthma Research and Innovation Partnership (EARIP) 2017 [cited 2017. Available from: https://www.asthma.org.uk/research/future/earip/.

2. Smith AD, Cowan JO, Filsell S, McLachlan C, Monti-Sheehan G, Jackson P, et al. Diagnosing asthma: comparisons between exhaled nitric oxide measurements and conventional tests. Am J Respir Crit Care Med. 2004;169(4):473-8.

3. Gibson P, Wang G, McGarvey L, Vertigan AE, Altman KW, Birring SS, et al. Treatment of Unexplained Chronic Cough: CHEST Guideline and Expert Panel Report. Chest. 2016;149(1):27-44.

4. Szefler SJ, Martin RJ, King TS, Boushey HA, Cherniack RM, Chinchilli VM, et al. Significant variability in response to inhaled corticosteroids for persistent asthma. J Allergy Clin Immunol. 2002;109(3):410-8.

5. Kelly HW, Nelson HS. Potential adverse effects of the inhaled corticosteroids. J Allergy Clin Immunol. 2003;112(3):469-78; quiz 79.

6. Powell H, Gibson PG. Inhaled corticosteroid doses in asthma: an evidence-based approach. Med J Aust. 2003;178(5):223-5.

7. Fahy JV. Type 2 inflammation in asthma--present in most, absent in many. Nat Rev Immunol. 2015;15(1):57-65.

8. Bjermer L, Alving K, Diamant Z, Magnussen H, Pavord I, Piacentini G, et al. Current evidence and future research needs for FeNO measurement in respiratory diseases. Respir Med. 2014;108(6):830-41.

9. Harnan SE, Tappenden P, Essat M, Gomersall T, Minton J, Wong R, et al. Measurement of exhaled nitric oxide concentration in asthma: a systematic review and economic evaluation of NIOX MINO, NIOX VERO and NObreath. Health Technol Assess. 2015;19(82):1-330.

10. Little SA, Chalmers GW, MacLeod KJ, McSharry C, Thomson NC. Non-invasive markers of airway inflammation as predictors of oral steroid responsiveness in asthma. Thorax. 2000;55(3):232-4. 11. Petsky HL, Kew KM, Turner C, Chang AB. Exhaled nitric oxide levels to guide treatment for adults with asthma. Cochrane Database Syst Rev. 2016;9:CD011440.

12. Agache I, Sugita K, Morita H, Akdis M, Akdis CA. The Complex Type 2 Endotype in Allergy and Asthma: From Laboratory to Bedside. Curr Allergy Asthma Rep. 2015;15(6):29.

13. Excellence NIfHaC. Measuring fractional exhaled nitric oxide concentration in asthma: NIOX MINO, NIOX VERO and NObreath 2013 [Available from: https://www.nice.org.uk/guidance/dg12/documents/measuring-fractional-exhaled-nitric-oxideconcentration-in-asthma-niox-mino-niox-vero-and-nobreath-diagnostics-consultation-document-pdfdocument2.

14. Report G. Global Strategy for Asthma Management and Prevention 2017 [Available from: http://ginasthma.org/.

15. Smith AD, Cowan JO, Brassett KP, Filsell S, McLachlan C, Monti-Sheehan G, et al. Exhaled nitric oxide: a predictor of steroid response. Am J Respir Crit Care Med. 2005;172(4):453-9.

16. Humbert M, Beasley R, Ayres J, Slavin R, Hebert J, Bousquet J, et al. Benefits of omalizumab as add-on therapy in patients with severe persistent asthma who are inadequately controlled despite best available therapy (GINA 2002 step 4 treatment): INNOVATE. Allergy. 2005;60(3):309-16.

17. GROUP TE. EQ-5D-3L User Guide. Basic information on how to use the EQ-5D-3L instrument 2015 [Available

from:

http://www.euroqol.org/fileadmin/user upload/Documenten/PDF/Folders Flyers/EQ-5D-

3L UserGuide 2015.pdf.

18. Malinovschi A, Van Muylem A, Michiels S, Michils A. FeNO as a predictor of asthma control improvement after starting inhaled steroid treatment. Nitric Oxide. 2014;40:110-6.

19. OpenClinica. [Available from: https://www.openclinica.com/clinical-trial-softwaresolutions/enterprise-edc-system/.

20. Solutions SP. [Available from: http://support.sas.com/software/93/.

21. Martin MJ, Wilson E, Gerrard-Tarpey W, Meakin G, Hearson G, McKeever TM, et al. The utility of exhaled nitric oxide in patients with suspected asthma. Thorax. 2016;71(6):562-4.

22. Spinou A, Birring SS. An update on measurement and monitoring of cough: what are the important study endpoints? J Thorac Dis. 2014;6(Suppl 7):S728-34.

23. American Thoracic S, European Respiratory S. ATS/ERS recommendations for standardized procedures for the online and offline measurement of exhaled lower respiratory nitric oxide and nasal nitric oxide, 2005. Am J Respir Crit Care Med. 2005;171(8):912-30.

24. C C-S. Assay Sensitivity. Wiley Encyclopedia of Clinical Trials: John Wiley \& Sons, Inc; 2008. 
25. Bayram M, Akkoyunlu ME, Kutbay Özçelik H, Karakose F, Yakar F, Sezer M, et al. The difference of FENO levels according to gender in healty subjects never smoked. European Respiratory Journal. 2014;44(Suppl 58).

26. Ko FW, Leung TF, Wong GW, Chu JH, Sy HY, Hui DS. Determinants of, and reference equation for, exhaled nitric oxide in the Chinese population. Eur Respir J. 2013;42(3):767-75.

27. Travers J, Marsh S, Aldington S, Williams M, Shirtcliffe P, Pritchard A, et al. Reference ranges for exhaled nitric oxide derived from a random community survey of adults. Am J Respir Crit Care Med. 2007;176(3):238-42.

28. Zhang H, Shu L, Cai X, Wang Z, Jiao X, Liu F, et al. Gender and age affect the levels of exhaled nitric oxide in healthy children. Exp Ther Med. 2013;5(4):1174-8.

29. Malinovschi A, Fonseca JA, Jacinto T, Alving K, Janson C. Exhaled nitric oxide levels and blood eosinophil counts independently associate with wheeze and asthma events in National Health and Nutrition Examination Survey subjects. J Allergy Clin Immunol. 2013;132(4):821-7 e1-5.

30. Agarwal R, Dhooria S, Aggarwal AN, Maturu VN, Sehgal IS, Muthu V, et al. Guidelines for diagnosis and management of bronchial asthma: Joint ICS/NCCP (I) recommendations. Lung India. 2015;32(Suppl 1):S3-S42.

31. Lucas AE, Smeenk FW, Smeele IJ, van Schayck CP. Overtreatment with inhaled corticosteroids and diagnostic problems in primary care patients, an exploratory study. Fam Pract. 2008;25(2):86-91.

32. Pandya D, Puttanna A, Balagopal V. Systemic effects of inhaled corticosteroids: an overview. Open Respir Med J. 2014;8:59-65.

33. Cane RS, Ranganathan SC, McKenzie SA. What do parents of wheezy children understand by "wheeze"? Arch Dis Child. 2000;82(4):327-32.

\section{Figures and Tables}

Table 1. Baseline characteristics of Per-Protocol set, by treatment arm

Mean (SD) for continuous variables; $\mathbf{n}(\%)$ for categorical variables*

Baseline study measure

Age (yrs)

Body Mass Index $\left(\mathrm{kg} / \mathrm{m}^{2}\right)$

Female

Smoking status

$$
\begin{array}{r}
\text { Current Smoker } \\
\text { Ex-Smoker }
\end{array}
$$

Never-Smoker

Country

UK
Singapore

FeNO (ppb)

$\mathrm{ACQ7}^{\dagger}$
Extrafine ICS $(\mathrm{N}=114)$

Placebo $(\mathrm{N}=100)$

$47.7(17.0)$

$27.6(6.0)$

$60(52.6 \%)$

$63(63.0 \%)$

$$
1(1.0 \%)
$$

$73(73.0 \%)$

$73(64.0 \%)$

$78(78.0 \%)$

$22(22.0 \%)$

$39.6(31.5)$

$1.71(0.6)$ 
EuroQol 5 Dimensions

Questionnaire

Clinical judgement: patient is

Clinical judgement: treatment is

${ }^{*}$ Variables were compared between treatment arms using Mann Whitney $U$ tests for continuous variables, and Chi-squared tests for categorical variables. A significant difference was observed for smoking status $(P=0.021) .{ }^{\dagger} A C Q 7$ recorded at either Visit 1 or Visit 2 of the study - original procedure was to collect at visit 2, but as many patients were found not to be eligible in terms of ACQ7, the protocol was changed to collect the baseline ACQ7 at visit 1, for efficiency. All other baseline measurements

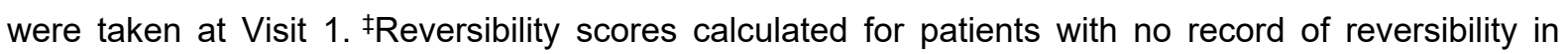
previous year (51 patients in extrafine ICS arm; 37 patients in placebo arm). Formula for reversibility: (FEV 1 post-salbutamol minus $\mathrm{FEV}_{1}$ pre-salbutamol)*100. ${ }^{\S}$ One patient in each arm had missing data for the clinical judgement questions. "Criteria: current/ex-smoker AND FEV $1 \%$ Predicted $<80 \%$ AND $\mathrm{FEV}_{1} / \mathrm{FVC}<0.7$. ICS=inhaled corticosteroids; $\mathrm{SD}=$ standard deviation; $\mathrm{FeNO}=$ fractional exhaled nitric oxide; $A C Q 7=a s t h m a$ control questionnaire, 7 questions; $F V_{1}=$ forced expiratory volume in first second; $\mathrm{FVC}=$ forced vital capacity; $\mathrm{PEF}=$ peak expiratory flow; $\mathrm{EQ}-5 \mathrm{D}-3 \mathrm{~L}=\mathrm{EuroQol} 5$ Dimensions Questionnaire; VAS=visual analogue scale. 
Figure 1. CONSORT flow diagram of study participation

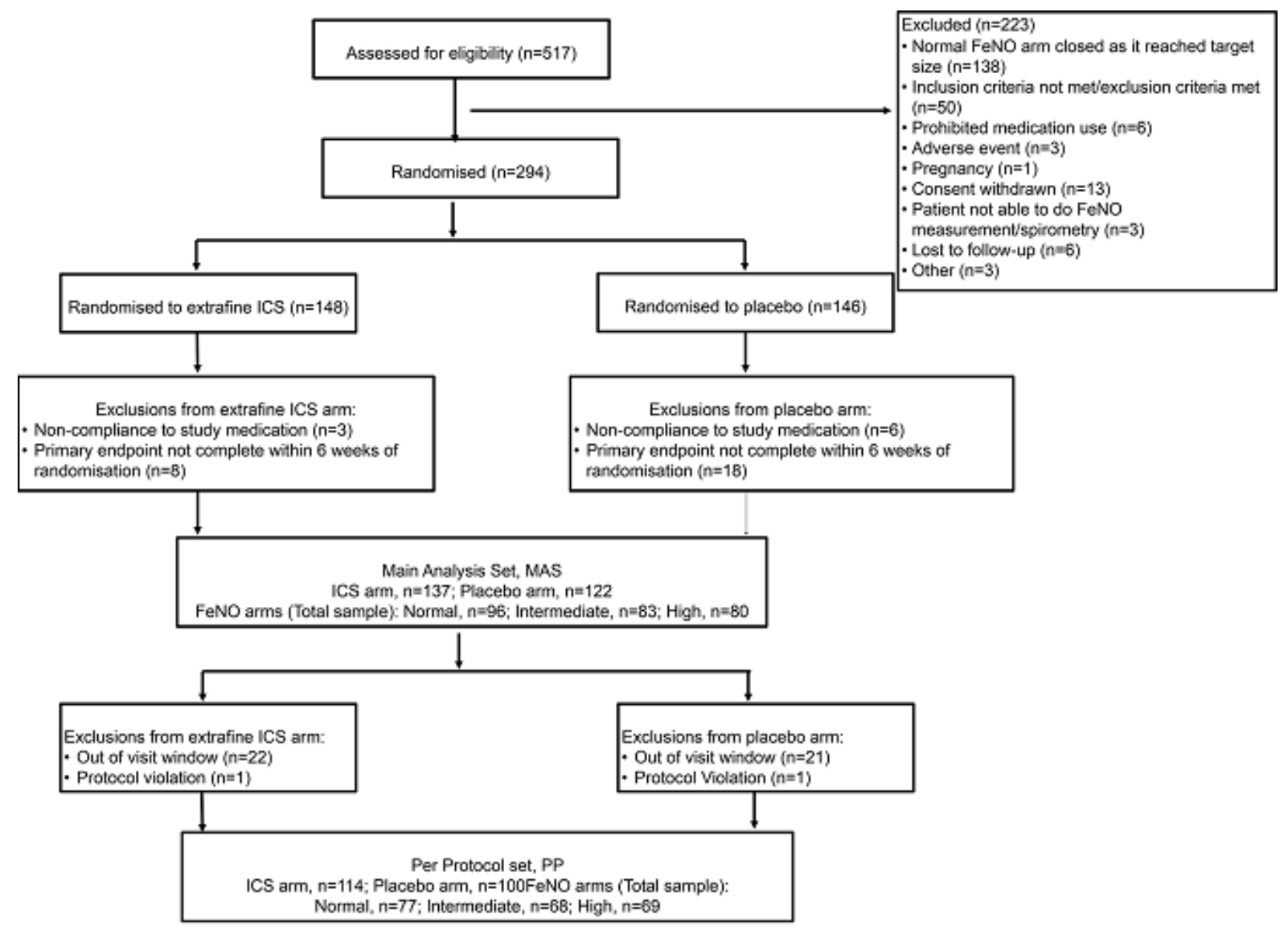

ICS=inhaled corticosteroids 
Table 2. Primary analysis: change in ACQ7 by treatment arm and baseline FeNO

\begin{tabular}{|c|c|c|c|}
\hline Measurement & $\begin{array}{l}\text { Extrafine } \\
\qquad \text { ICS } \\
(\mathrm{N}=114)\end{array}$ & $\begin{array}{l}\text { Placebo } \\
(\mathrm{N}=100)\end{array}$ & $\begin{array}{c}\text { Adjusted treatment effect } \\
\text { (extrafine ICS minus placebo) / } \\
\text { Interaction effect with } \mathrm{FeNO}^{\dagger}\end{array}$ \\
\hline \multicolumn{4}{|l|}{$\begin{array}{l}\text { Mean (SD) chan } \\
\text { minus baseline): }\end{array}$} \\
\hline & & 0.56 & \\
\hline Total $(n=214)$ & $0.82(0.70)$ & $(0.78)$ & $0.29(0.08,0.49)$ \\
\hline & & 0.73 & \\
\hline Normal FeNO ${ }^{\ddagger}(n=77)$ & $0.80(0.59)$ & $(0.62)$ & $0.10(-0.24,0.44)$ \\
\hline & & 0.47 & \\
\hline Intermediate $\mathrm{FeNO}^{\ddagger}(\mathrm{n}=68)$ & $0.70(0.75)$ & $(0.89)$ & $0.25(-0.10,0.61)$ \\
\hline & & 0.49 & \\
\hline High FeNO ${ }^{\ddagger}(n=69)$ & $0.97(0.77)$ & $(0.78)$ & $0.49(0.14,0.84)$ \\
\hline
\end{tabular}

Interaction effect:

Difference $(95 \% \mathrm{Cl})$ in treatment effect ${ }^{\dagger}$

for every 10ppb increase in baseline

FeNO

$0.071(0.002,0.139)^{\pi}$

\section{SENSITIVITY ANALYSIS}

Difference in Gradients (Extrafine ICS -

Placebo)

$0.045(0.006,0.084)^{7}$

*All models adjusted by baseline $\mathrm{FeNO}$ and smoking status. ${ }^{\dagger}$ Difference in treatment effect is interpreted as the additional change in continuous outcome in the extrafine ICS versus placebo arm when baseline FeNO is $10 \mathrm{ppb}$ higher. ${ }^{\ddagger}$ Normal, $\leq 25 \mathrm{ppb}$; Intermediate, $>25 \mathrm{ppb}-<40 \mathrm{ppb}$; High, $\geq 40 \mathrm{ppb}$. ${ }^{\top} \mathrm{P}$ value $=0.044$; Significant $p$ values indicate a significant interaction, i.e. baseline $\mathrm{FeNO}$ is associated with the size of the treatment response. $\mathrm{Cl}=$ confidence interval; $\mathrm{FeNO}=$ fractional exhaled nitric oxide; $\mathrm{ACQ} 7=$ asthma control questionnaire, 6 questions and $\mathrm{FEV}_{1} .{ }^{\sharp} \mathrm{A}$ negative value indicates lower improvement in outcome as FeNO increases for Extrafine ICS compared to Placebo. 
Table 3 Patients with improvement in ACQ ( $\geq 0.5)$

\begin{tabular}{|c|c|c|c|c|c|c|c|c|}
\hline \multirow[t]{2}{*}{$\begin{array}{l}\text { Change in ACQ } \\
\text { (Per-protocol population) }\end{array}$} & \multicolumn{2}{|c|}{$\begin{array}{l}\text { Normal } \\
(\mathrm{n}=77)\end{array}$} & \multicolumn{2}{|c|}{$\begin{array}{l}\text { Intermediate } \\
\qquad(\mathrm{n}=68)\end{array}$} & \multicolumn{2}{|c|}{$\begin{array}{l}\text { High } \\
(n=69)\end{array}$} & \multicolumn{2}{|c|}{$\begin{array}{c}\text { Total } \\
(n=214)\end{array}$} \\
\hline & $\begin{array}{c}\text { Extra Fine ICS } \\
(n=45)\end{array}$ & $\begin{array}{c}\text { Placebo } \\
(n=32)\end{array}$ & $\begin{array}{c}\text { Extra Fine ICS } \\
(n=33)\end{array}$ & $\begin{array}{c}\text { Placebo } \\
(n=35)\end{array}$ & $\begin{array}{c}\text { Extra Fine ICS } \\
(n=36)\end{array}$ & $\begin{array}{c}\text { Placebo } \\
(n=33)\end{array}$ & $\begin{array}{c}\text { Extra Fine ICS } \\
(n=114)\end{array}$ & $\begin{array}{l}\text { Placebo } \\
(n=100)\end{array}$ \\
\hline Clinical improvement $(\geq 0.5)^{*}$ & $32(71.1 \%)$ & $22(68.8 \%)$ & $21(63.6 \%)$ & $18(51.4 \%)$ & $27(75.0 \%)$ & $18(54.6 \%)$ & $80(70.2 \%)$ & $58(58.0 \%)$ \\
\hline $\begin{array}{l}\text { No clinically important change } \\
(<0.5 \text { and } \geq-0.5)\end{array}$ & $13(28.9 \%)$ & $9(28.1 \%)$ & $8(24.24 \%)$ & $11(31.4 \%)$ & $9(25.0 \%)$ & $11(33.33 \%)$ & $30(26.3 \%)$ & $31(31.0 \%)$ \\
\hline Clinical deterioration $(<-0.5)$ & $0(0.0 \%)$ & $1(3.1 \%)$ & $4(12.12 \%)$ & $6(17.1 \%)$ & $0(0.0 \%)$ & $4(12.12 \%)$ & $4(3.5 \%)$ & $11(11.0 \%)$ \\
\hline Number-needed-to-treat (NNT) & \multicolumn{2}{|l|}{58.82} & \multicolumn{2}{|l|}{33.33} & \multicolumn{2}{|l|}{17.24} & \multicolumn{2}{|l|}{34.42} \\
\hline
\end{tabular}


Table 4. Primary analysis: change in ACQ6 by treatment arm and baseline FeNO

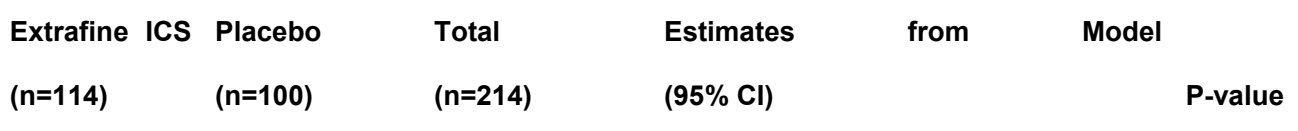




\begin{tabular}{|c|c|c|c|c|c|}
\hline & $\begin{array}{l}\text { Extrafine ICS } \\
(n=114)\end{array}$ & $\begin{array}{l}\text { Placebo } \\
(n=100)\end{array}$ & $\begin{array}{l}\text { Total } \\
(n=214)\end{array}$ & $\begin{array}{l}\text { Estimates } \\
(95 \% \mathrm{Cl})\end{array}$ & $\begin{array}{l}\text { Model } \\
\qquad \text { P-value }\end{array}$ \\
\hline \multicolumn{6}{|l|}{ Per-Protocol Set } \\
\hline $\mathrm{n}(\%$ non-missing) & $114(83.2 \%)$ & $100(82.0 \%)$ & $214(82.6 \%)$ & & \\
\hline Mean (SD) & $0.93(0.81)$ & $0.65(0.90)$ & $0.80(0.86)$ & $0.29(0.08,0.49) \ddagger$ & \\
\hline Median (IQR) & $0.8(0.5,1.5)$ & $0.8(0.1,1.2)$ & $0.8(0.3,1.3)$ & & \\
\hline Min, Max & $-1.0,4.2$ & $-1.7,2.7$ & $-1.7,4.2$ & & \\
\hline \multicolumn{6}{|c|}{ Estimated change in ACQ improvement when baseline FeNO is $10 \mathrm{ppb}$ higher } \\
\hline Extrafine ICS & & & & $0.053(-0.005,0.112)$ & \\
\hline Placebo & & & & $-0.022(-0.076,0.031)$ & \\
\hline Difference in Gradients (Extrafine ICS - Placebo) & & & & $0.076(-0.004,0.155)$ * & 0.061 \\
\hline
\end{tabular}




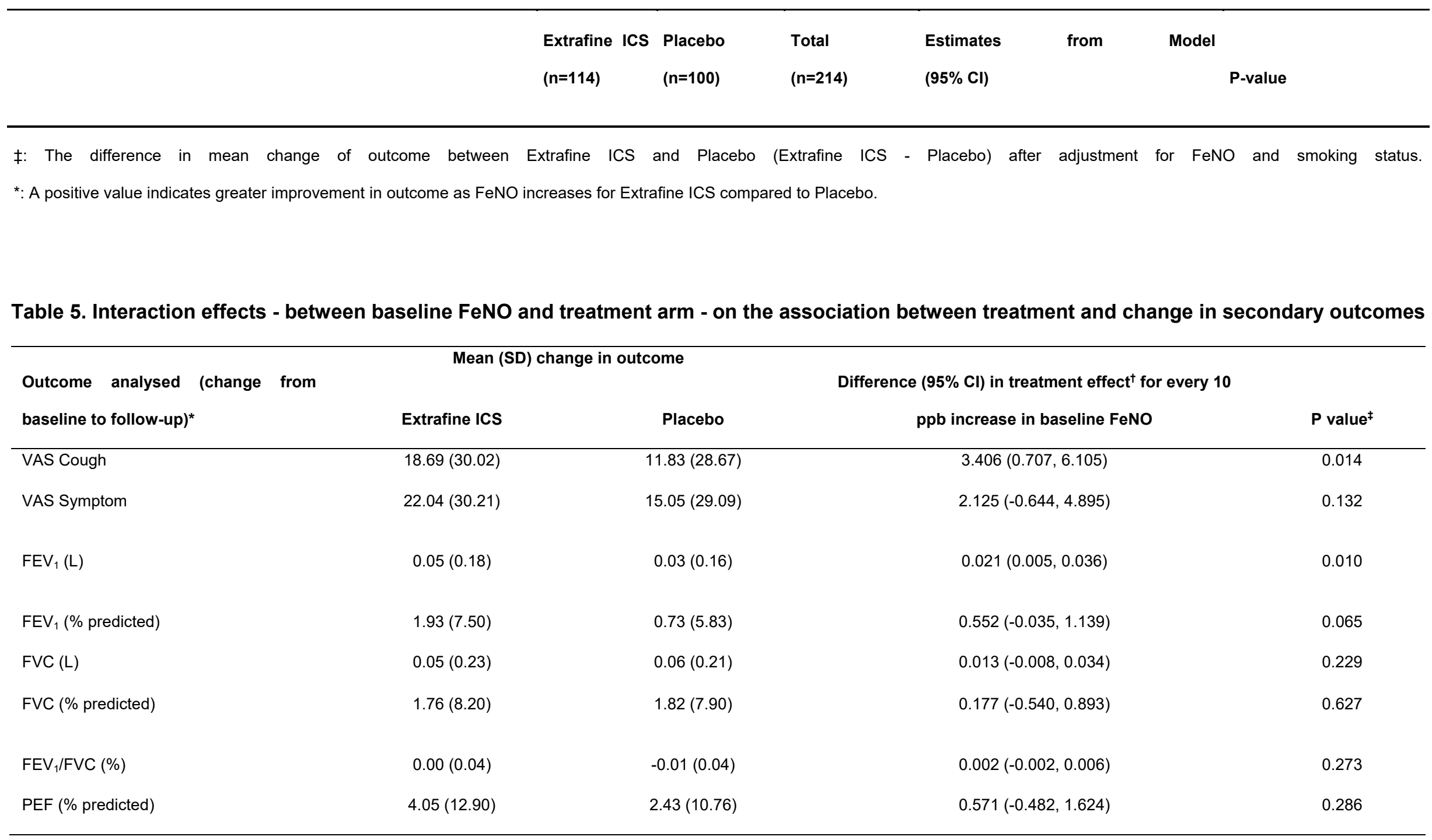


*Models adjusted by baseline FeNO and smoking status. ${ }^{\dagger}$ Difference in treatment effect is interpreted as the additional change in continuous outcome in the extrafine ICS versus placebo arm when baseline FeNO is $10 \mathrm{ppb}$ higher. $¥$ Significant $\mathrm{p}$ values indicate a significant interaction, i.e. baseline FeNO is associated

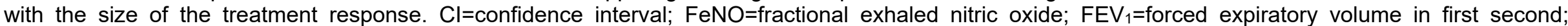
$F V C=$ forced vital capacity; $P E F=$ peak expiratory flow. VAS=visual analogue scale. Severity of Cough (VAS) was measured on a 0-100 vertical visual analogue scale for severity of cough 0 (no cough) 100 (worse cough ever). Perception of Asthma symptoms (VAS) was measured on a 0-100 horizontal visual analogue scale for how bothersome they find their asthma 0 (not at all bothersome) 100 (extremely bothersome).

\section{Table 6. Summary of adverse events during the study, in all randomised patients $(n=294)$}

\begin{tabular}{|c|c|c|c|c|c|c|c|c|}
\hline \multirow[b]{2}{*}{ Summary measure } & \multicolumn{3}{|c|}{ Extrafine ICS } & \multicolumn{3}{|c|}{ Placebo } & \multirow{2}{*}{$\begin{array}{c}\text { Extrafine ICS } \\
\quad(n=148)\end{array}$} & \multirow{2}{*}{ Placebo $(n=146)$} \\
\hline & $\begin{array}{c}\text { Normal FeNO* } \\
(n=56)\end{array}$ & $\begin{array}{l}\text { Intermediate } \\
\text { FeNO* }(n=44)\end{array}$ & $\begin{array}{l}\text { High FeNO* } \\
(n=48)\end{array}$ & $\begin{array}{c}\text { Normal FeNO* } \\
(n=51)\end{array}$ & $\begin{array}{l}\text { Intermediate } \\
\text { FeNO* }(n=48)\end{array}$ & $\begin{array}{c}\text { High FeNO* } \\
(n=47)\end{array}$ & & \\
\hline $\begin{array}{l}\text { Number of patients } \\
\text { with at least } 1 \text { adverse } \\
\text { event }\end{array}$ & $13(23.2 \%)$ & $15(34.1 \%)$ & $19(39.6 \%)$ & $17(33.3 \%)$ & $18(37.5 \%)$ & $20(42.6 \%)$ & $47(31.8 \%)$ & $55(37.7 \%)$ \\
\hline \multicolumn{9}{|l|}{$\begin{array}{l}\text { Number of adverse } \\
\text { events, by severity }{ }^{\dagger}\end{array}$} \\
\hline Mild & 12 & 19 & 17 & 14 & 19 & 20 & 48 & 53 \\
\hline Moderate & 3 & 3 & 13 & 10 & 10 & 10 & 19 & 30 \\
\hline Severe & 0 & 0 & 0 & 0 & 0 & 2 & 0 & 2 \\
\hline Total & 15 & 22 & 30 & 24 & 29 & 32 & 67 & 85 \\
\hline $\begin{array}{llr}\text { Number of adverse } \\
\text { events due to } \\
\text { treatment }\end{array}$ & 0 & 4 & 5 & 4 & 8 & 3 & 9 & 15 \\
\hline $\begin{array}{l}\begin{array}{l}\text { Number of adverse } \\
\text { events } \\
\text { discontinuation of ousing } \\
\text { study drug }\end{array} \\
\end{array}$ & 0 & 0 & 3 & 6 & 2 & 9 & 3 & 17 \\
\hline
\end{tabular}

some limitation of usual activities; Severe; Inability to carry out usual activities 
Figure 2. Predictors of clinical response in ACQ7 and VAS cough, in the extrafine ICS arm of the exploratory analysis set ( $n=137)$

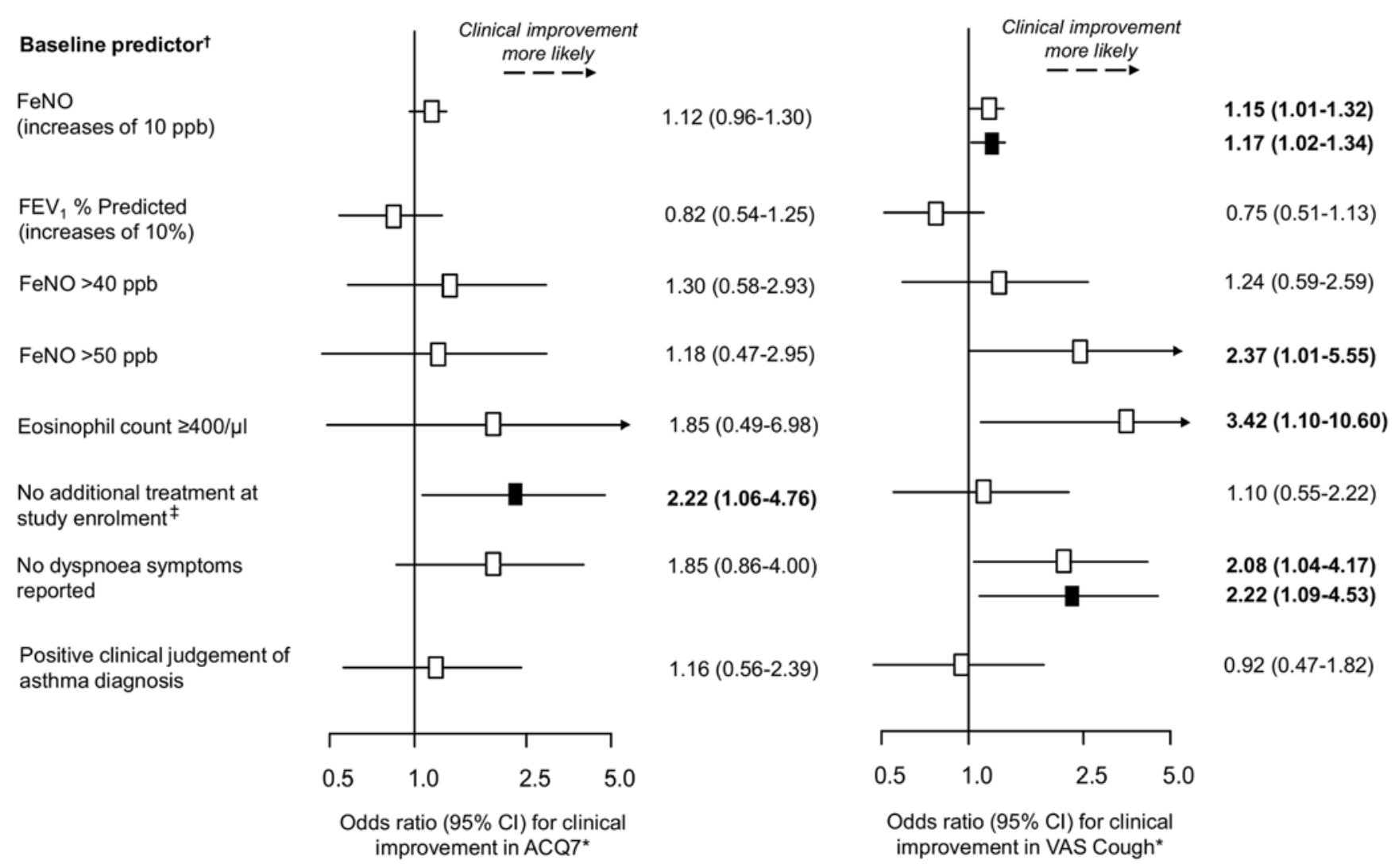

Unadjusted $\mathbf{A d j u s t e d} /$ retained in final model

${ }^{*}$ Clinical improvement defined as decrease $\geq 0.5$ for ACQ7, and decrease $\geq 20 \mathrm{~mm}$ for VAS Cough. ${ }^{\dagger}$ All variables, except continuous FeNO and FEV $1 \%$ Predicted, are binary. The reference category for odds ratios is the opposite category of what is presented, e.g. odds for patients reporting no dyspnoea symptoms are compared with odds for patients who did report dyspnoea symptoms. Odds ratio for continuous FeNO represents the increase in odds for every $10 \mathrm{ppb}$ increase in baseline FeNO. ${ }^{\ddagger}$ Additional treatment defined as any treatment received for non-specific respiratory symptoms at study enrolment, apart from extrafine ICS or any treatment listed in the exclusion criteria. Bold text indicates significant association. $\mathrm{Cl}=$ confidence interval; FeNO=fractional exhaled nitric oxide; VAS=visual analogue scale. 